Revista Brasileira de Meteorologia, v.25, n.3, 295 - 310, 2010

\title{
OS ÚLTIMOS AVANÇOS NA PREVISIBILIDADE DOS CAMPOS DE UMIDADE NO SISTEMA GLOBAL DE ASSIMILAÇÃO DE DADOS E PREVISÃO NUMÉRICA DE TEMPO DO CPTEC/ INPE
}

\author{
LUIZ FERNANDO SAPUCCI, DIRCEU LUIS HERDIES, RITA VALÉRIA ANDREOLI DE SOUZA, \\ JOÃO GERD ZELL DE MATTOS, JOSÉ ANTÔNIO ARAVÉQUIA.
}

Instituto Nacional de Pesquisas Espaciais (INPE) Centro de Previsão de Tempo e Estudos Climáticos (CPTEC). Cachoeira Paulista - SP, Brasil. \{luiz.sapucci;dirceu.herdies;joão.gerd;araveq\}@cptec.inpe.br; rasouza@uea.edu.br

Recebido Agosto 2008 - Aceito Fevereiro 2010

\begin{abstract}
RESUMO
Nos últimos anos, duas importantes implementações foram feitas no sistema global de assimilação de dados e previsão de tempo do CPTEC/INPE, as quais têm uma relação direta com a previsibilidade dos campos de umidade. A primeira é a assimilação de valores do vapor d'água integrado na atmosfera (IWV-Integrated Water Vapor). A segunda é a utilização de uma versão melhorada do modelo de previsão de tempo, juntamente com o aumento da resolução da grade. O objetivo do presente estudo é caracterizar o impacto de tais implementações na melhoria da previsibilidade da umidade. Para isso, quatro experimentos foram realizados utilizando as versões T126L28 (sem as melhorias) e T213L42 (com a implementação das melhorias) do modelo global do CPTEC, com e sem a assimilação do IWV. Os resultados obtidos mostram que o impacto da assimilação de IWV é maior nas primeiras horas de previsão. Por outro lado, as melhorias implementadas no modelo geram bons resultados para as previsões de médio (após 60 horas) a longo prazo. Em consequência disso, o impacto combinado de ambas as implementações é significativo ao longo de todo o período de integração avaliado. Nessa circunstância, o intervalo das previsões do IWV consideradas estatisticamente válidas (coeficiente de correlação de anomalia acima de 0.6) é ampliado em 25 horas na região tropical e em 18 horas na América do Sul.
\end{abstract}

Palavras chave: Assimilação de dados; IWV; PSAS; AIRS-AMSU; PNT.

\begin{abstract}
LATEST ADVANCES IN THE PREDICTION OF HUMIDITY FIELDS IN THE DATA ASSIMILATION AND NUMERICAL WEATHER PREDICTION GLOBAL SYSTEM FROM CPTEC/INPE

Two important implementations, directly related to the prediction of atmospheric humidity fields, have been introduced during in the recent years in the weather forecast model and its data assimilation system at CPTEC/INPE. The first is the inclusion of Integrated Water Vapor (IWV) data in the assimilation scheme. The second is the improvement of the weather forecast model along with an increase in the grid resolution. The goal of this paper is to characterize the impact of these implementations in the prediction of humidity fields. To achieve this goal four experiments were conducted using the T126L28 (without the improvement) and T213L42 (with the improvement) versions of the CPTEC's global model, with and without assimilation of IWV. The results show that the impact of IWV assimilation is larger in the first hours of forecast. On the other hand, the larger impacts produced by the improvements in the weather forecast model occur from the $60^{\text {th }} \mathrm{h}$ of forecast onward. Consequently the combined impact of both implementations represent an improvement throughout the integration period or the period at which predictions of IWV present an acceptable skill (above 60\%) were extended in up to 25 hours over the global tropical regions and up to 18 hours over South America.
\end{abstract}

Keywords: Data assimilation; IWV; PSAS; AIRS-AMSU; NWP. 


\section{INTRODUÇÃO}

O vapor d'água presente na atmosfera desempenha um importante papel na circulação geral atmosférica, sendo responsável pelo transporte de boa parte da energia da superfície para os níveis mais elevados, absorvendo o calor latente de evaporação na superfície e liberando-o durante o processo de formação de nuvens. Nas regiões tropicais, onde o gradiente horizontal de temperatura é pequeno, esse processo é a principal fonte de energia (Holton, 1992). A advecção do vapor d'água e a liberação do calor latente influenciam a instabilidade vertical, a estrutura e a evolução de sistemas convectivos atmosféricos. Nessas áreas, o erro dos modelos de Previsão Numérica de Tempo (PNT) em representar os parâmetros hidrológicos é relativamente elevado, o que limita o uso dos mesmos para o entendimento do ciclo hidrológico e seu papel na variabilidade climática da umidade na atmosfera. Hou et al. (2000) demonstraram que a assimilação de observações relacionadas com a umidade na atmosfera pode reduzir significativamente esses erros.

A característica mais relevante da umidade na atmosfera é sua alta variabilidade temporal e espacial, podendo em um curto período de tempo apresentar oscilações significativas na sua localização (Randel et al., 1996). Diversos processos físicos e químicos estão associados à sua concentração. Entre outros processos pode-se citar a evaporação de superfícies aquáticas, a evapotranspiração dos seres vivos (em especial a vegetação), a ocorrência de fenômenos atmosféricos (como orvalho, chuvas e tempestades) e a circulação atmosférica (em especial os processos convectivos). Devido a essa alta variabilidade temporal, o desempenho da PNT em prever os campos de umidade para médio e longo prazo é relativamente baixo. Os valores do Coeficiente de Correlação da Anomalia (CCA), estatística frequentemente utilizada para medir o desempenho de um modelo de PNT, para as variáveis associadas à umidade caem, em média, para valores inferiores a 0.6 após as primeiras 36 horas de previsão. Esse valor de 0.6 no CCA é considerado um indicador limite da validade das previsões (Krishnamurti et al., 2003). Assim, as previsões dos campos de umidade são efêmeras, possuem vida útil relativamente curta. Portanto, todas e quaisquer melhorias na previsibilidade dos campos de umidade são relevantes, pois podem impactar diretamente na qualidade final da previsão de tempo operacional.

No Centro de Previsão de Tempo e Estudos Climáticos do Instituto Nacional de Pesquisas Espaciais (CPTEC/ INPE), adotou-se duas novas implementações na assimilação operacional, as quais têm um impacto direto na melhoria da previsibilidade dos campos de umidade. A primeira é a assimilação de valores do vapor d'água integrado na coluna atmosférica (IWV-Integrated Water Vapor) e a outra é a utilização de uma versão melhorada do modelo de previsão de tempo juntamente com o aumento da resolução de sua grade.

No que se refere à assimilação do IWV, sabe-se que na assimilação de dados o objetivo principal é obter um estado atmosférico mais realístico e o seu sucesso está diretamente relacionado com a quantidade e a qualidade de observações atmosféricas disponíveis. Para o caso da umidade, a dependência de observações de qualidade bem distribuídas espacialmente é intensificada pela alta variabilidade do vapor d'água atmosférico. Na literatura há diversos trabalhos que reportam resultados positivos da assimilação de valores do IWV. Kuo et al. (1993), a partir de um método de assimilação baseado em relaxação newtoniana (nudging) (Stauffer e Seamen, 1990), mostrou que a assimilação de valores do IWV provenientes de uma rede de radiômetros corrige a estrutura vertical da umidade e melhora a previsão de precipitação de curto prazo. Utilizando diferentes fontes de dados, outros pesquisadores apresentaram resultados que evidenciam a importância da assimilação do IWV. Uma descrição detalhada dos métodos utilizados para a obtenção de valores do IWV de diferentes fontes de observações, como radiossondas, receptores GPS, radiômetros e satélites sondadores de umidade, é apresentada por Sapucci et al. (2007). Ledvina e Pfaendtner (1995) mostraram uma redução significativa do viés da umidade nas análises geradas com a assimilação de valores de IWV do SSM/I no sistema de assimilação do Goddard Space Flight Center (GEOS-1 DAO). Com a assimilação de valores do IWV, provenientes de redes de receptores GPS instaladas nos EUA no National Oceanic and Atmospheric Administration (NOAA) Forecast Systems Laboratory, foi obtido um impacto positivo na previsão de umidade de curto prazo (Smith et al., 2000). Trabalhos mais recentes têm utilizado longas séries de dados das densificações das redes de receptores GPS sobre os EUA e resultados importantes têm sido obtidos. Gutman et al. (2004) utilizando esse tipo de dado mostram que o impacto da assimilação dos mesmos no desempenho do modelo tem crescido ao longo dos anos acompanhando o processo de densificação da rede de receptores. Smith et al. (2007) mostram que o impacto da assimilação de valores do IWV varia sazonalmente, em função da localização geográfica e do ciclo diário da umidade. Um importante resultado desse estudo é a operacionalização da assimilação desses dados tanto na NOAA como no National Centers for Environmental Prediction (NCEP), ocorrida em junho de 2006, visando obter operacionalmente a comprovada melhoria nas previsões de curto prazo da umidade. No Physical-space Statistical Analysis System (PSAS) (Cohn et al., 1998), que atualmente é o sistema de assimilação de dados operacional no CPTEC, encontra-se implementada uma rotina que permite a assimilação do IWV. Baseado nos resultados 
apresentados em Sapucci et al. (2010), os valores do IWV provenientes do sensor AIRS/AMSU (Atmospheric InfraRed Sounder/Advanced Microwave Sounding Unit) passaram a ser assimilados operacionalmente na versão global do PSAS desde março de 2007.

O desenvolvimento de novas tecnologias de processamento de dados ocorrido nos últimos anos tem ampliado significativamente a capacidade computacional dos centros de previsão. Isso tem viabilizado a utilização de modelos de PNT com parametrizações mais sofisticadas e com maior resolução e, consequentemente, melhorado o detalhamento espacial das variáveis prognósticas utilizadas. No CPTEC/INPE isso tem ocorrido tanto na versão global dos modelos numéricos quanto na regional. Na versão global atualmente operacional houve implementações de novas funcionalidades e parametrizações físicas, além da modernização de todo o sistema (Tomita et al., 2006). O objetivo principal dessa modernização foi aumentar o desempenho computacional do modelo. Isso possibilitou que, com o mesmo recurso computacional disponível, uma maior resolução pudesse ser utilizada, passando da resolução T126L28 (aproximadamente 100x100km na horizontal e 28 níveis na vertical) para a resolução T213L42 (aproximadamente 60x60 km e 42 níveis na vertical). Com a utilização de modelos mais sofisticados e de maior resolução obtém-se uma melhoria na capacidade dos modelos em caracterizar melhor os campos de umidade, tornado-os mais sensíveis às oscilações espaciais dessa variável.

Considerando os pontos destacados acima, o presente trabalho tem por objetivo mostrar o impacto destas implementações na melhoria da previsibilidade dos campos de umidade gerados pelo sistema global de assimilação e previsão de tempo do CPTEC-INPE. Para isso, foram realizados quatro experimentos: um, utilizando a versão do modelo sem as melhorias, na resolução T126L28, e sem a assimilação do IWV; um segundo, com o mesmo modelo e resolução, mas com a assimilação do IWV; um terceiro, utilizando a versão melhorada na resolução T213L42 e sem a assimilação do IWV, e um último, com o mesmo modelo do terceiro experimento, porém com a assimilação do IWV. Na próxima seção são apresentados os detalhes relevantes associados às melhorias implementadas nessa nova versão do modelo global do CPTEC e a assimilação de valores do IWV. Detalhes dos dados utilizados, dos experimentos realizados e a metodologia de avaliação empregada, são apresentados na seção 3. Na seção 4 são apresentados os resultados obtidos a partir da comparação dos quatro experimentos realizados. Na seção 5 é apresentada uma análise dos resultados ao avaliar o impacto da assimilação do IWV e das melhorias presentes na nova versão do modelo, bem como a ação conjunta dessas duas implementações. Na seção 6 são apresentados os comentários finais e as conclusões.

\section{IMPLEMENTAÇÕES VISANDO À MELHORIADA PREVISIBILIDADE DOS CAMPOS DE UMIDADE}

A eficiência do Modelo de Circulação Geral Atmosférica (MCGA) em gerar previsões confiáveis, depende do desempenho do sistema de assimilação em representar da forma mais fiel possível o estado da atmosfera em um dado instante (Kalnay, 2003), e do próprio modelo em representar adequadamente as equações que governam o sistema como um todo. Mas reproduzir fielmente a distribuição da umidade como é encontrada na natureza, ainda é um grande desafio por dois motivos principais. O primeiro é a necessidade de modelos globais de alta resolução e o segundo é a disponibilidade de dados de umidade com cobertura global e uniformemente distribuídos. Ambos os motivos estão associados à alta variabilidade espacial do vapor d'água na atmosfera, a qual requer sistemas de assimilação de dados que permitam a utilização maciça de dados de satélites sondadores de umidade, e modelos eficientes na parametrização física dos processos que envolvem essa variável atmosférica. Levando ao limite, os modelos precisariam resolver os processos físicos sem o uso de parametrizações dos complicados fenômenos que envolvem a radiação, a convecção e os processos na superfície. No entanto, isso não é possível devido à capacidade computacional ainda insuficiente para tal, bem como também pela carência de conhecimento científico mais preciso a respeito. Com o aumento da quantidade de dados assimilados, o tempo de processamento para que o sistema de assimilação encontre uma solução apropriada cresce consideravelmente. Em consequência disso, há uma demanda significativa pelo aumento da capacidade computacional nos centros de previsão de tempo. Esse aumento na capacidade computacional pode ser traduzido em melhoria do desempenho dos processadores (execução de um maior número de operações em menor tempo), envolvendo eficientes processos de troca de mensagens entre processadores (MPI e OpenMP, por exemplo), e na capacidade de armazenamento de dados (espaço em disco). Seguindo uma tendência mundial, no CPTEC/INPE tem havido um significativo aumento da capacidade computacional com a aquisição de um novo supercomputador escalar com 1024 processadores, e a futura aquisição de outro supercomputador vetorial com maior capacidade de processamento. Essa ampliação permite que o centro vislumbre a possibilidade de se utilizar operacionalmente sistemas de assimilação de dados e modelos de previsão com parametrizações físicas mais sofisticadas e com maior resolução, contribuindo para se obter uma melhor previsibilidade dos campos de umidade. 


\subsection{Modernizações Implementadas no Modelo Global de PNT do CPTEC.}

Entre a versão anterior do modelo global de PNT do CPTEC (Kinter et al., 1997) e a mais atual (Tomita et al., 2006), as modernizações realizadas podem ser divididas em três partes: reestruturação do código, implementação de novas parametrizações e aumento da resolução.

O objetivo do processo de reestruturação do código iniciado em 2002 é disponibilizar novas funcionalidades e melhorar o desempenho computacional, não apenas em máquinas de processamento vetorial como em máquinas escalares. A intenção é disponibilizar uma versão que também considere o crescimento das novas tecnologias de supercomputadores de baixo custo, como os clusters de PCs (Tomita et al., 2006). Para isso, todo o sistema foi reestruturado em módulos independentes e maciçamente paralelizado para memória MPI (message passing interface) e compartilhada OpenMP.

Com relação às novas parametrizações, o modelo modernizado contempla dois métodos de dinâmica espectral: uma versão espectral Euleriana computacionalmente mais eficiente e a Semi-lagrangeana. Uma implementação importante presente na nova versão é a possibilidade de se utilizar diferentes números de pontos da grade de longitude por latitude em função da latitude. Essa implementação foi denominada grade reduzida, a qual proporciona uma redução dos cálculos nos pontos próximos ao polo sem degradar a previsibilidade do modelo. Embora a física seja a mesma utilizada na versão anterior (Cavalcanti et al., 2002), na nova versão estão disponíveis novos esquemas de convecção e radiação. Para convecção profunda foi implementado o esquema de Grell (Grell, 1993). Para convecção rasa está disponível o esquema de Souza (Souza, 1999). Para a radiação de onda curta foi implementado o esquema CLIRAD-SW (Chou e Suarez, 1996).

O terceiro ponto a ser destacado, e o mais importante do ponto de vista da assimilação de medidas de umidade, dada sua alta variabilidade espacial, é o aumento da resolução do modelo ocorrido com a implementação dessa nova versão. Com o acoplamento do sistema de assimilação PSAS, uma maior resolução do modelo permite que um maior número de pontos da grade do modelo seja corrigido pelas observações, quando o sistema de assimilação combina as previsões de curto prazo, usualmente de 6 horas (definidas como First Guess e doravante denominada FG) com as observações ao gerar a análise. Isso se dá pelo fato de que cada tipo de observação tem uma área de ação (definida por um raio) e a intensidade com que os pontos da grade do modelo são corrigidos pelas observações é proporcional à distância destes ao ponto de observação. Em consequência disso, resoluções maiores permitem que um maior número de pontos esteja dentro dessa área de atuação e, além disso, tendem a colocar os pontos da grade mais próximos dos pontos de observação e, portanto melhores corrigidos por elas. Como o vapor d'água atmosférico tem alta variabilidade espacial, os benefícios gerados pelas menores distâncias entre os pontos de observação e os pontos da grade do modelo são amplificados para as observações da umidade na atmosfera.

\subsection{Assimilação do IWV na PNT}

A mais confiável fonte de informação da umidade na atmosfera é proveniente das medidas realizadas pelas radiossondas, pois as realizam de forma direta e empregam técnicas sofisticadas, além de fornecerem a melhor resolução vertical (Sapucci et al., 2005). No entanto, estes dados estão concentrados sobre os continentes e observa-se uma lacuna na disponibilidade dos mesmos tanto na resolução temporal como espacial. Os lançamentos operacionais das radiossondas são, em geral, efetuados duas vezes ao dia, e se concentram, em sua maioria, no Hemisfério Norte. Além do número de lançamentos ser inadequado, essa configuração espacial não privilegia a vasta região oceânica que cobre o globo, em especial no Hemisfério Sul. É evidente que apenas as radiossondagens não são suficientes para caracterizar adequadamente a distribuição global de umidade, e que outras fontes dessa informação, como os satélites sondadores de umidade, devem ser exploradas.

No processo de assimilação de dados, objetiva-se obter uma estimativa ótima do estado da atmosfera, de forma que a representação desse estado seja a mais realista possível, ao combinar observações com o FG. Tal estimativa é considerada ótima no sentido de que o "erro da estimativa" seja minimizado. No processo de assimilação há o operador de observação responsável por interpolar os dados do modelo para o ponto de observação e transformar as grandezas físicas contidas no FG, tais como componentes zonal e meridional do vento, altura geopotencial, pressão, temperatura e umidade específica em grandezas físicas observadas. Com a implementação de operadores apropriados, é possível utilizar observações não convencionais como perfis de radiância (satélites sondadores), refratividade derivadas das observações do sistema GPS (Global Positioning System) (Bevis et al., 1992) e perfis do raio de ocultação e ângulo de curvatura provenientes da radiocultação GPS (Anthes et al., 2008), entre outras observações.

Para a assimilação dos valores do IWV, há a necessidade de um procedimento adicional, pois como se trata de um valor integrado, tal observação está diretamente relacionada não apenas com uma observação, mas com todos os valores do perfil de umidade do FG. Em tal procedimento o valor integrado é convertido em um perfil de umidade explorando sua relação direta com o perfil de umidade específica. $\mathrm{Na}$ metodologia clássica aplicada a esse processo, a ideia básica 
é fazer com que os valores do IWV inicializados tendam aos valores observados. Para isso, os valores do perfil de umidade específica do modelo são modificados, enquanto é mantida a distribuição vertical da umidade nesse perfil (Kuo et al., 1993). Essa metodologia foi apresentada inicialmente por Gal-Chen et al. (1986) na assimilação de outros valores integrados, e depois por Kuo et al. (1993) aplicado aos valores do IWV. Este último trabalho foi pioneiro na assimilação dessa variável. A mesma está implementada no sistema de assimilação PSAS, o qual além da concentração de umidade do FG considera também parâmetros, que definem pesos para os diferentes níveis do perfil vertical de umidade. O benefício em potencial com o uso desses parâmetros é que o mesmo permite alterar a intensidade com que os valores do IWV modificam os valores de umidade específica entre os diferentes níveis de pressão. O ponto mais crítico com relação à assimilação do IWV no PSAS é que, nesse caso, um processamento adicional específico para a umidade é necessário. Como o código do PSAS não é adequadamente paralelizado, o custo computacional das rodadas com a assimilação do IWV torna-se relativamente elevado. Cada ciclo do PSAS com assimilação do IWV na versão T213, tem demorado em média 3,5 horas. Esse foi um fator limitante na definição do período em que as diferentes rodadas experimentais realizadas nesse trabalho foram feitas.

\section{DETALHES DOS EXPERIMENTOS REALIZADOS, DOS DADOS UTILIZADOS E DO MÉTODO DE AVALIAÇÃO.}

O sistema de assimilação utilizado foi o PSAS (versão 2003) e o modelo global de PNT utilizado foi o do CPTECCOLA (Kinter et al., 1997; Cavalcanti et al., 2002) nas resoluções T126L28 e T213L42. Enquanto que na versão T213L42 foram implementadas as melhorias discutidas na seção 2.1, na versão T126L28 tais melhorias não estão presentes. As versões T126L28 e T213L42 aqui utilizadas são semelhantes às versões operacionais, sendo a primeira até junho 2005 e a segunda depois dessa data até o presente. As diferenças entre essas versões podem ser sintetizadas pelas informações apresentadas na Tabela 1. As rodadas dos diferentes experimentos foram realizadas a partir de cada um dos horários sinóticos, 00, 06, 12 e 18 h UTC, e para cada integração do modelo foram feitas previsões de até 114 horas. O período escolhido para esse estudo foi o mês de abril de 2007 por se tratar de um mês intrassazonal e, portanto, de mais difícil previsibilidade. As diferentes rodadas são referenciadas pelas seguintes denominações:

- T126semIWV: PSAS com o MCGA na resolução T126L28 sem assimilação do IWV;

- T126comIWV: PSAS com o MCGA na resolução T126L28 com assimilação do IWV;

- T213semIWV: PSAS com o MCGA contemplando as melhorias discutidas na seção 2.1, na resolução T213L42, sem assimilação do IWV;

- T213comIWV: PSAS com o MCGA contemplando as melhorias discutidas na seção 2.1, na resolução T213L42, com assimilação do IWV.

Os valores do IWV considerados nesse estudo são provenientes de sondagens do sensor AIRS/AMSU a bordo do satélite AQUA. No processamento dos dados coletados por esse sensor foi utilizada a versão 4 do algoritmo da National Aeronautics and Space Administration (NASA) (Susskind et al., 2003). Considerando alguns indicadores de qualidade produzidos nesse processo, foram descartados valores com qualidade degradada, e para aumentar a eficiência do processo de assimilação foi aplicado um filtro para reduzir a quantidade de dados utilizados. As passagens do satélite AQUA sobre a América do Sul estão concentradas as 06 e 18 UTC. A Figura 1 mostra a distribuição espacial dos valores do IWV provenientes do sensor AIRS/AMSU disponíveis para a assimilação.

Em todos os quatro experimentos, além dos dados do IWV incluídos ou não, foram assimilados valores de altura geopotencial provenientes de perfis de temperatura medidos

Tabela 1 - Configurações utilizadas nas diferentes resoluções do modelo de PNT do CPTEC.

\begin{tabular}{ccc}
\hline \hline Itens configurados & Resolução T126L28 & Resolução T213L42 \\
\hline Dinâmica Espectral & Euleriano & Euleriano otimizado \\
Representação em ponto de grade & Não reduzida & Reduzida \\
Convecção profunda & Kuo & Kuo \\
Convecção rasa & Tiedtke & Grell \\
Radiação de onda longa & Hashvardhan & Souza \\
Parametrização de superficie & Ssib & Ssib \\
Parametrização da camada limite & Mellor e Yamada & Mellor e Yamada \\
\hline \hline
\end{tabular}



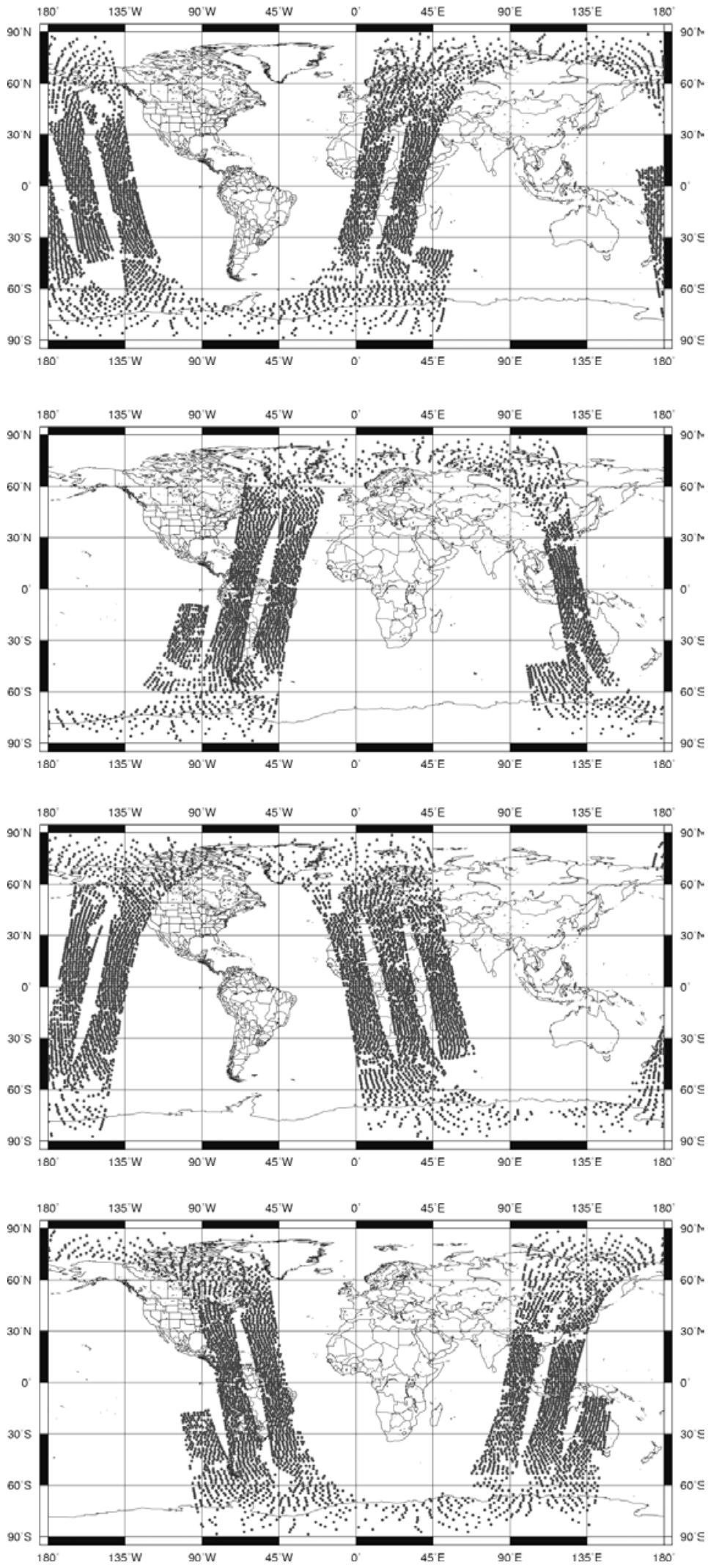

Figura 1 - Distribuição espacial típica dos valores do IWV provenientes do sensor AIRS/AMSU para os diferentes horários em que os experimentos foram rodados. 
por satélites NOAA, do tipo ATOVS [Advanced TIROS (Television Infrared Observation Satellite) Operational Vertical Sounder] (Reale, 2002) e do satélite AQUA (sensor AIRS/AMSU); Medidas das componentes zonal e meridional do vento sobre os oceanos, provenientes do satélite Titan II, missão QuikScat (Nasa's Quik Scatterometer); Valores de altura geopotencial, temperatura, pressão, umidade específica, vento zonal e meridional provenientes de sensores instalados em navios, aviões, bóias oceânicas e em estações meteorológicas de superfície e ar superior, disponíveis no GTS (Global Telecommunication System). Tais dados são provenientes de processos operacionais no CPTEC/INPE e, portanto, a quantidade utilizada aqui é semelhante à disponível nas rodadas operacionais.

Na avaliação da melhoria da previsibilidade dos campos de umidade foram calculados os valores do CCA para as variáveis diretamente associadas ao vapor d'água atmosférico. Essa estatística foi escolhida porque mede o desempenho do modelo ao avaliar a correlação entre a anomalia de um campo da previsão e uma anomalia de um campo da análise, sem levar em consideração a magnitude do campo e sim sua coerência espacial (Krishnamurti et al., 2003). Para o cálculo das anomalias são utilizados campos de médias mensais climatológicas geradas a partir das análises do NCEP no período de 12 anos (1987-1998). Embora não possam ser consideradas a verdade, tais análises são boas aproximações se avaliado o grande número de dados utilizados em sua elaboração, e por isso servem para os propósitos do presente estudo. Os valores do CCA são calculados para diferentes níveis do modelo de forma independente, aplicando a seguinte fórmula:

$$
C C A=\frac{\sum_{i=1}^{I} \sum_{j=1}^{J}\left[\left(\alpha_{i, j}^{P}-\alpha_{i, j}^{C}\right) \cdot\left(\alpha_{i, j}^{A}-\alpha_{i, j}^{C}\right)\right]}{\left\{\left[\sum_{i=1}^{I} \sum_{j=1}^{J}\left(\alpha_{i, j}^{P}-\alpha_{i, j}^{C}\right)^{2}\right]\left[\sum_{i=1}^{I} \sum_{j=1}^{J}\left(\alpha_{i, j}^{A}-\alpha_{i, j}^{C}\right)^{2}\right]\right\}^{1 / 2}}
$$

na qual o símbolo $\alpha$ representa uma variável qualquer, os índices A, P e C representam as análises, as previsões e a climatologia, respectivamente. Os índices I e J representam o número total de pontos de grade nas direções latitudinal e longitudinal, respectivamente.

Na avaliação as variáveis escolhidas foram: o IWV e a umidade relativa em diferentes níveis de pressão $(925 \mathrm{hPa}$, $850 \mathrm{hPa}, 700 \mathrm{hPa}, 500 \mathrm{hPa}, 300 \mathrm{hPa}$ e $250 \mathrm{hPa}$ ). Embora nas camadas acima de $500 \mathrm{hPa}$ a umidade apresente-se reduzida, análises em 300 e 250 hPa são apresentadas devido ao forte impacto na radiação e, por consequência, nas previsões de mais longo prazo. Enquanto os valores do IWV, por serem valores integrados, podem fornecer informações da melhoria global da previsibilidade do perfil de umidade, os valores de umidade relativa permitem avaliar o impacto dessas melhorias em diferentes camadas da atmosfera. Nessa avaliação, três diferentes regiões foram consideradas: Hemisfério Sul (HS), entre $80^{\circ} \mathrm{S}-$ $20^{\circ} \mathrm{S}$; região tropical (RT), entre $20^{\circ} \mathrm{S}-20^{\circ} \mathrm{N}$; e América do Sul (AS), entre $50^{\circ} \mathrm{S}-10^{\circ} \mathrm{N}$ e $80^{\circ} \mathrm{W}-30^{\circ} \mathrm{W}$. As análises utilizadas como referência são as provenientes dos experimentos sem a assimilação do IWV para as diferentes versões do modelo. As séries temporais do CCA foram calculadas a partir das previsões geradas nas rodadas dos diferentes experimentos a partir de cada um dos horários sinóticos, 00, 06, 12 e 18 h UTC, para todo o mês de abril de 2007. Valores médios para as primeiras 6 horas de integração foram calculados para cada uma das variáveis
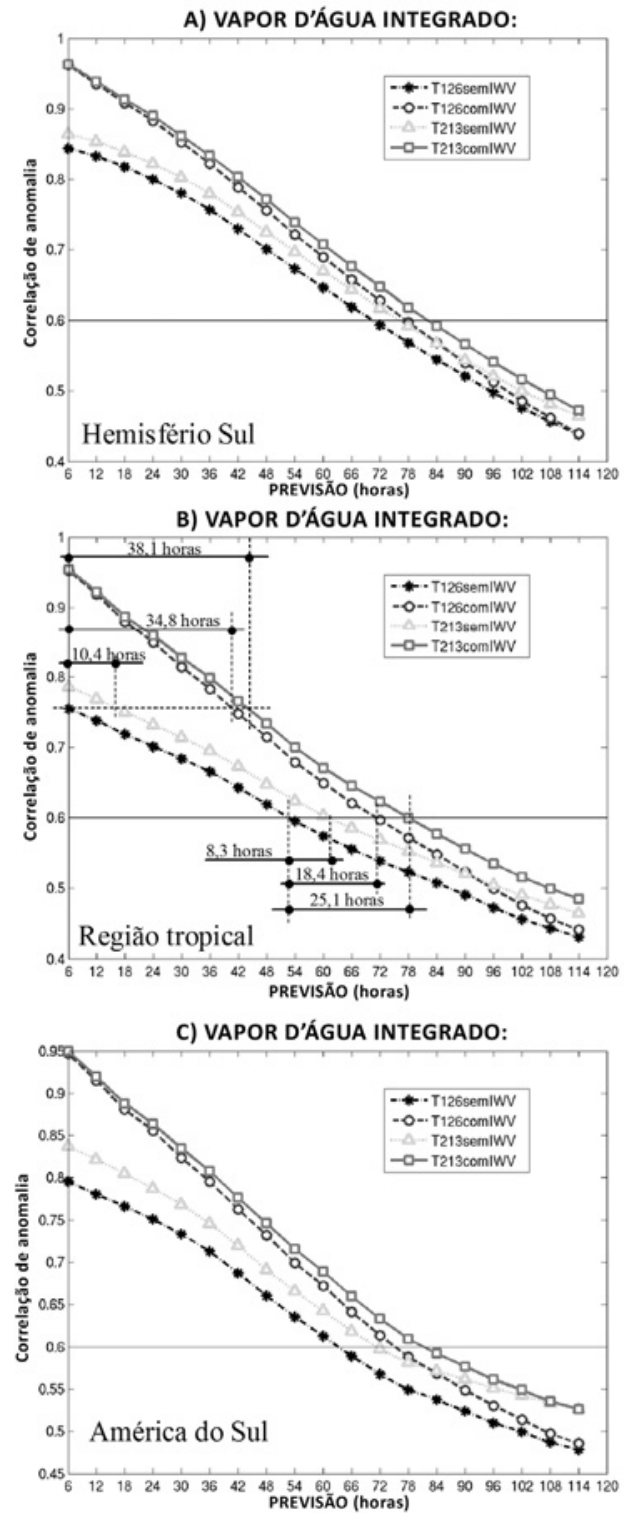

Figura 2 - Valores do Coeficiente da Correlação de Anomalia (CCA) do IWV obtidos nos diferentes experimentos realizados em função do tempo de previsão para o (a) Hemisfério Sul, (b) região tropical e (c) América do Sul. 
consideradas e nas diferentes regiões já mencionadas. Depois para 12 horas de integração, e sucessivamente até 114 horas.

\section{RESULTADOS}

Na Figura 2 são apresentados os valores do CCA do IWV obtidos nos diferentes experimentos em função do tempo de previsão para (a) HS, para (b) RT e (c) AS. Observa-se nessa figura que as diferenças entre os valores do CCA são mais significativas na região tropical e na América do Sul do que no Hemisfério Sul. Por esse motivo, as regiões RT e AS serão focadas na análise dos valores da umidade relativa.
Nas Figuras 3 e 4 são apresentados os valores do CCA da umidade relativa obtidos nos diferentes experimentos em função do tempo de previsão em (a) 925 hPa, (b) 850 hPa, (c) 700 hPa, (d) 500 hPa, (e) 300 hPa e (f) 250 hPa, para a região tropical e para a América do Sul, respectivamente.

Um resultado importante e que deve ser destacado na comparação entre os valores do CCA gerados nas diferentes rodadas avaliadas (Figuras 2, 3 e 4), é a extensão das previsões estatisticamente aceitáveis (valores do CCA maiores que 0.6). Daqui para frente, o intervalo de tempo de integração do modelo em que as previsões geradas podem ser consideradas estatisticamente aceitáveis é denominado a)

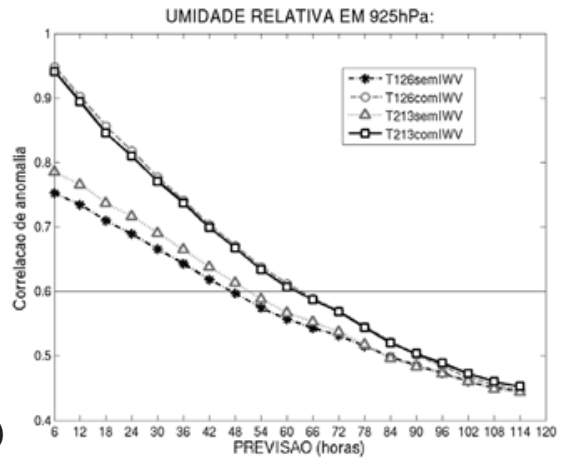

c)
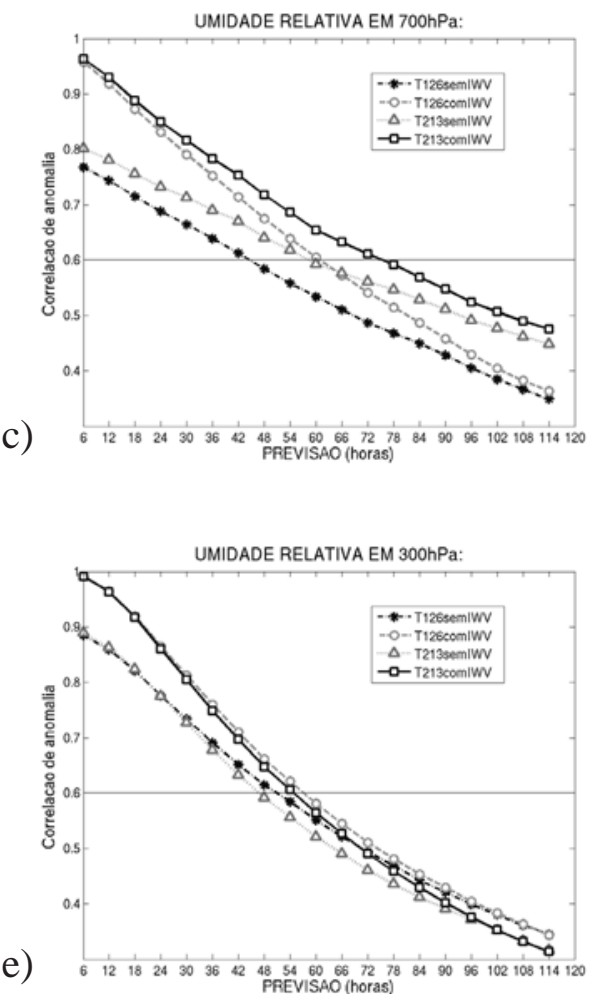
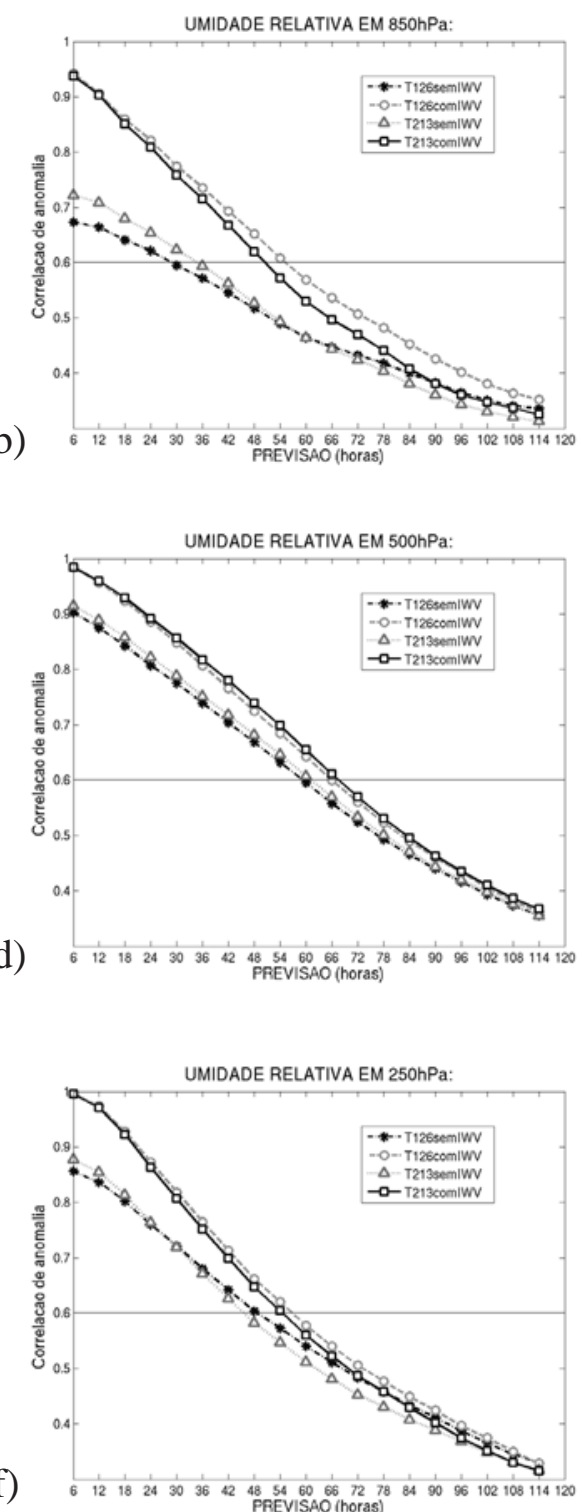

Figura 3 - Valores do Coeficiente da Correlação de Anomalia (CCA) da umidade relativa obtidos nos diferentes experimentos realizados em função do tempo de previsão para a região tropical em (a) 925 hPa, (b) 850 hPa, (c) 700 hPa, (d) 500 hPa, (e) 300 hPa e (f) 250 hPa. 
extensão das previsões válidas. Há um traço horizontal no limiar de 0.6 em todos os gráficos das Figuras 2, 3 e 4, para evidenciar a extensão das previsões válidas. Observa-se que, de forma geral, com as implementações efetuadas houve uma ampliação desse intervalo. Como pode ser notada na Figura 2b, na rodada T126semIWV, a última previsão considerada estatisticamente aceitável, ou válida, é de 48 horas. No experimento T126comIWV observa-se que as previsões de 66 horas, também podem ser consideradas válidas, ou seja, com a assimilação do IWV houve uma ampliação da extensão das previsões válidas de 18 horas. Caso seja considerado o experimento T213semIWV, observa-se que as melhorias do modelo geram uma ampliação de 8 horas na extensão das previsões válidas. Mas, considerado-se o experimento a)
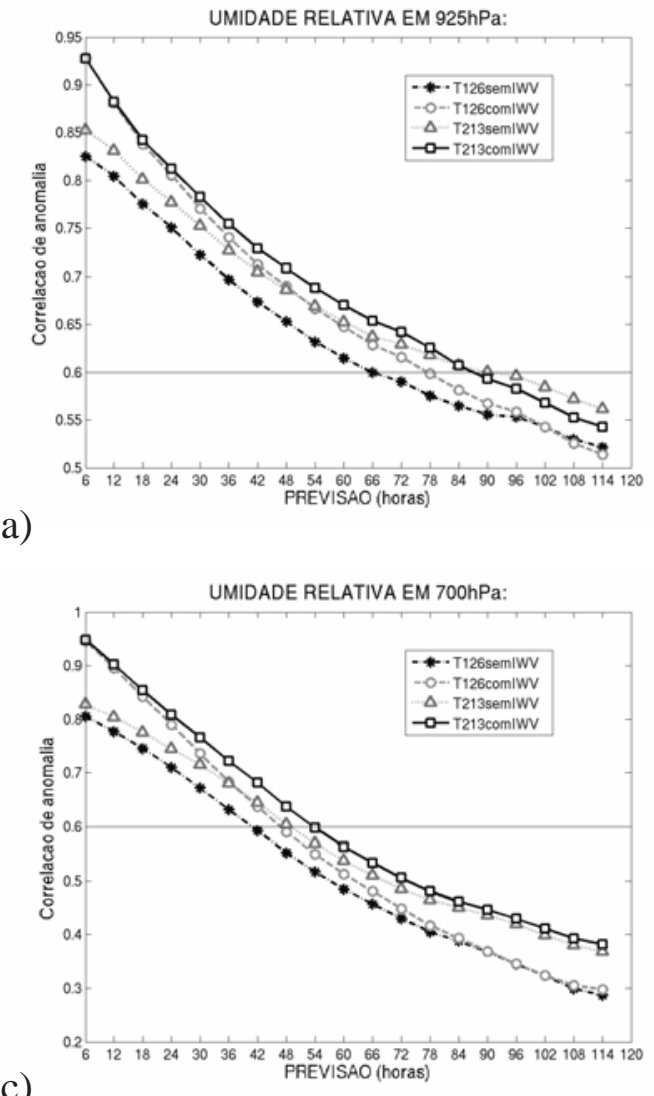

C)

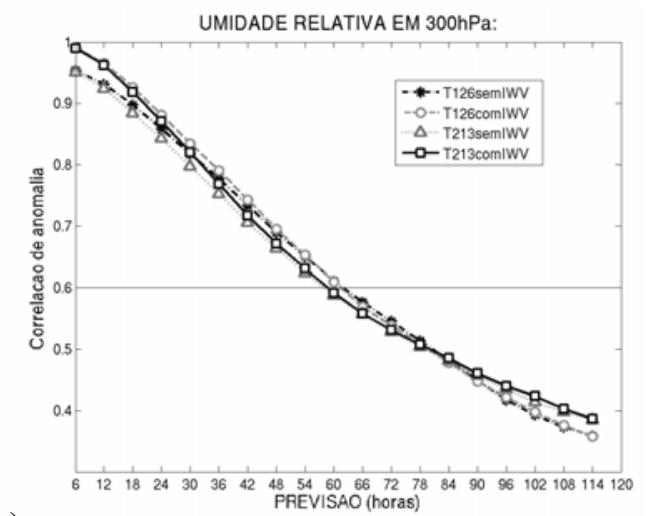

e)

Figura 4 - Valores do Coeficiente da Correlação de Anomalia (CCA) da umidade relativa obtidos nos diferentes experimentos realizados em função do tempo de previsão para a região tropical em (a) 925 hPa, (b) 850 hPa, (c) 700 hPa, (d) 500 hPa, (e) 300 hPa e (f) 250 hPa.

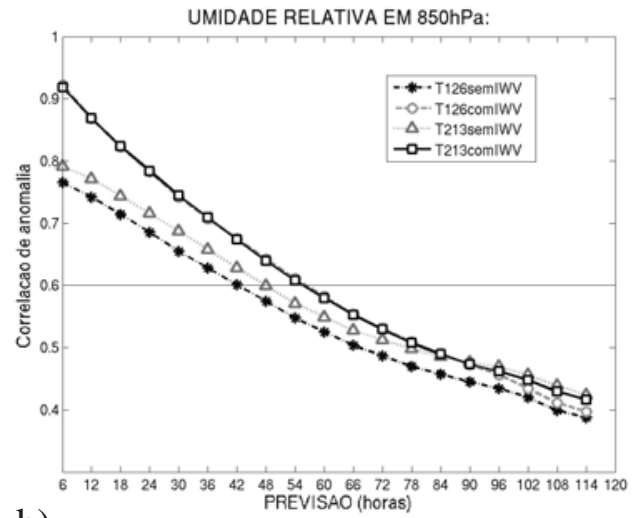

b)

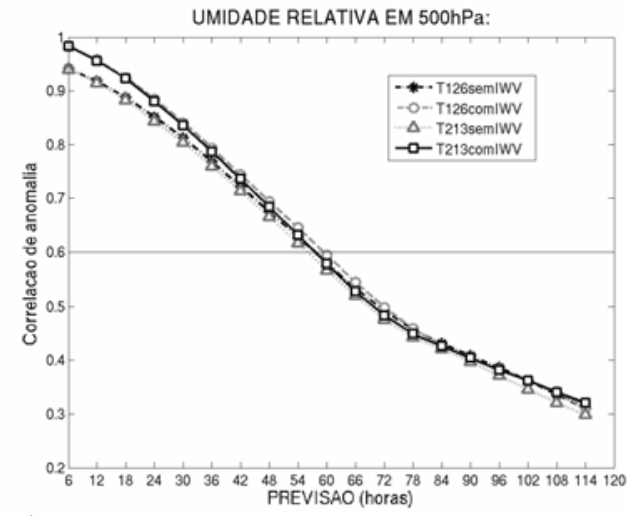

d)

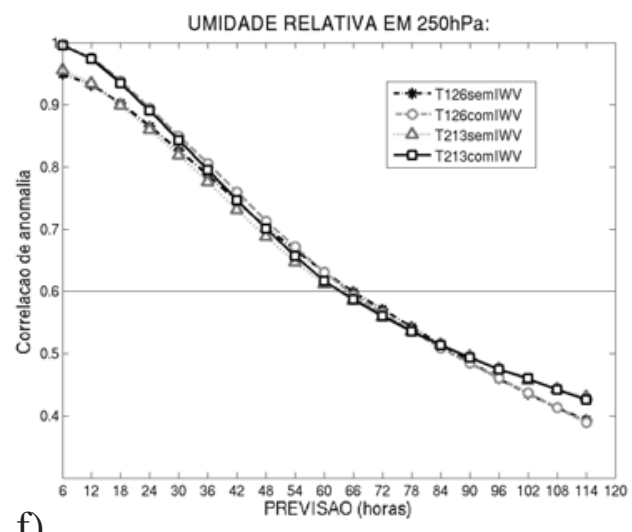

f) 
T213comIWV, ou seja, as melhorias do modelo e a assimilação do IWV simultaneamente, essa ampliação passa a ser de 25 horas. Tal ampliação pode ser considerada um aumento da capacidade do modelo de PNT em prever o comportamento de uma determinada variável. A Tabela 2 mostra os valores da ampliação da extensão das previsões válidas obtidas com a assimilação do IWV na versão T126L28, ao comparar os experimentos T126comIWV com o T126semIWV. O mesmo foi feito na versão T213L42 ao comparar os experimentos T213comIWV com o T213semIWV. Nessa tabela são mostrados também os resultados obtidos com as melhorias do modelo sem assimilar o IWV, ao comparar os experimentos T213semIWV com T126semIWV, bem como na assimilação do IWV ao comparar os experimentos T213comIWV com o T126comIWV. Os resultados da ação conjunta de ambas as implementações foram calculados a partir da comparação dos experimentos T213comIWV com o T126semIWV. Os valores apresentados nessa tabela foram calculados algebricamente a partir dos pontos pertencentes às curvas com valores iguais a 0.6 e se referem às regiões HS, RT e AS.

Além da ampliação da extensão das previsões válidas, um outro resultado a ser destacado é o aumento da correlação de anomalia para as previsões de 6 horas. Melhorias na qualidade desses campos podem trazer uma contribuição bastante positiva para a qualidade final da análise após um período de assimilação de dados, uma vez que são utilizados ciclicamente como FG da rodada seguinte. O cálculo desse ganho é feito considerando o ponto pertencente à curva dos valores do CCA do experimento avaliado, que apresente o mesmo valor da CCA do FG do experimento de referência. Esse resultado é ilustrado na Figura 2b, na qual observa-se que o experimento T126comIWV gerou um ganho no CCA do FG de 34,8 horas. Em outras palavras, pode-se dizer que a correlação de anomalia do FG obtida sem incluir o IWV é similar às previsões de 40 horas geradas no experimento em que o IWV foi assimilado. A Tabela 3 apresenta os valores do ganho em horas no CCA da umidade no FG obtidos com a assimilação do IWV, nas versões T126L28 e T213L42, com as melhorias do modelo, assimilando ou não o IWV e com a ação conjunta de ambas as implementações para o HS, RT e AS. De forma similar aos cálculos feitos para gerar os resultados apresentados na Tabela 2, os valores apresentados na Tabela 3 também foram calculados algebricamente e os mesmos pares de experimentos foram utilizados.

Tabela 2 - Expansão da extensão das previsões válidas (CCA acima de 0.6) obtida com a assimilação do IWV, com as melhorias no modelo e com a ação conjunto de ambas as implementações para o HS, RT e AS (valores em horas).

\begin{tabular}{|c|c|c|c|c|c|c|c|c|}
\hline \multirow{2}{*}{\multicolumn{2}{|c|}{ Implementações }} & \multicolumn{7}{|c|}{ Variáveis } \\
\hline & & \multirow{2}{*}{$\begin{array}{l}\text { IWV } \\
7.0\end{array}$} & \multirow{2}{*}{$\begin{array}{c}\mathrm{UR} \\
925 \mathrm{hPa} \\
0.0\end{array}$} & \multirow{2}{*}{$\begin{array}{c}\mathrm{UR} \\
850 \mathrm{hPa} \\
2.6\end{array}$} & \multirow{2}{*}{$\begin{array}{c}\mathrm{UR} \\
700 \mathrm{hPa} \\
4.5\end{array}$} & \multirow{2}{*}{$\begin{array}{c}\mathrm{UR} \\
500 \mathrm{hPa} \\
0.5\end{array}$} & \multirow{2}{*}{$\begin{array}{c}\mathrm{UR} \\
300 \mathrm{hPa} \\
0.3\end{array}$} & \multirow{2}{*}{$\begin{array}{c}\mathrm{UR} \\
250 \mathrm{hPa} \\
0.0\end{array}$} \\
\hline \multirow{3}{*}{$\begin{array}{l}\text { Assimilação } \\
\text { do IWV (no } \\
\text { T126L28) }\end{array}$} & HS & & & & & & & \\
\hline & $\mathrm{RT}$ & 18.4 & 16.0 & 26.4 & 16.3 & 6.7 & 6.3 & 8.1 \\
\hline & AS & 12.0 & 11.6 & 14.0 & 5.8 & 1.9 & -0.5 & -0.8 \\
\hline \multirow{3}{*}{$\begin{array}{l}\text { Assimilação } \\
\text { do IWV (no } \\
\text { T213L42) }\end{array}$} & HS & 6.1 & 0.0 & 0.0 & 3.9 & 1.3 & 0.4 & 1.2 \\
\hline & RT & 16.7 & 11.4 & 14.9 & 16.4 & 6.3 & 5.0 & 7.8 \\
\hline & AS & 10.1 & -2.6 & 7.8 & 4.9 & 1.7 & 1.5 & 0.8 \\
\hline \multirow{3}{*}{$\begin{array}{l}\text { Melhorias do } \\
\text { modelo (não } \\
\text { assimilando } \\
\text { o IWV) }\end{array}$} & HS & 5.6 & 0.0 & 0.0 & 21.9 & 3.9 & 4.7 & 4.1 \\
\hline & RT & 8.4 & 4.5 & 6.3 & 14.0 & 2.2 & -1.7 & -3.9 \\
\hline & AS & 8.5 & 24.7 & 5.6 & 7.9 & -1.6 & -3.3 & -4.2 \\
\hline \multirow{3}{*}{$\begin{array}{l}\text { Melhorias do } \\
\text { modelo } \\
\text { (assimilando } \\
\text { o IWV) }\end{array}$} & HS & 4.7 & 0.0 & 8.3 & 21.3 & 4.6 & 4.9 & 2.5 \\
\hline & RT & 6.7 & -1.0 & -4.8 & 14.6 & 1.6 & -2.3 & -2.3 \\
\hline & AS & 6.1 & 9.7 & -0.6 & 6.9 & -1.8 & -2.7 & -1.7 \\
\hline \multirow{3}{*}{$\begin{array}{l}\text { Melhorias do } \\
\text { modelo e } \\
\text { assimilação } \\
\text { do IWV }\end{array}$} & HS & 11.7 & 0.0 & 10.9 & 25.7 & 5.9 & 5.3 & 2.5 \\
\hline & RT & 25.1 & 15.0 & 21.6 & 30.9 & 8.3 & 3.9 & 5.9 \\
\hline & AS & 18.1 & 21.3 & 13.4 & 12.8 & 0.1 & -3.2 & -2.5 \\
\hline
\end{tabular}




\section{ANÁLISE DOS RESULTADOS}

Os resultados apresentados na seção anterior são analisados em três etapas. A primeira visa avaliar o impacto da assimilação dos valores do IWV na previsibilidade da umidade, tanto na versão T126L28, como na nova versão do modelo com as melhorias implementadas. Para isso, os resultados obtidos nos experimentos T126comIWV e T126semIWV são comparados, bem como nos experimentos T213comIWV e T213semIWV. Na segunda, o enfoque é a avaliação do impacto das melhorias presentes na versão T213L42 na previsibilidade dos campos de umidade. Nessa etapa os resultados dos experimentos T126semIWV e T213semIWV são comparados para que seja acessado o impacto dessas melhorias na ausência da assimilação do IWV. Por outro lado, os experimentos T126comIWV e T213comIWV são comparados para se verificar o impacto das melhorias do modelo, quando o IWV é assimilado. Na terceira e última etapa é avaliado o impacto da ação conjunta da assimilação do IWV e das melhorias implementadas no modelo na previsibilidade da umidade. Para isso, os resultados gerados nos experimentos T126semIWV e T213comIWV são comparados.

\subsection{Impacto da Assimilação do IWV na Previsibilidade dos Campos de Umidade}

Comparando os resultados apresentados pelos experimentos com e sem a assimilação do IWV (Figuras 2, 3 e 4), tanto na versão T126L28 do modelo, como na versão com as novas implementações, observa-se que existem alguns padrões que podem ser claramente identificados. Com relação aos valores do IWV (Figura 2), dois padrões se destacam:

- O impacto da assimilação do IWV é mais significativo para as primeiras horas de previsão e decai com o tempo de integração do modelo. Enquanto que para as previsões de 6 horas o CCA é significativamente maior (passando de 0.75 para 0.95 na versão T126L28 sobre a região tropical), para previsões de 114 horas a diferença tende a zero. Uma consequência desse padrão é que o impacto da assimilação do IWV apresenta um ganho maior na qualidade da previsão de $6 \mathrm{~h}$, o que pode ser associado diretamente a um maior ganho na qualidade do FG, do que na expansão das previsões válidas (veja Tabelas 2 e 3);

Tabela 3 - Ganho no CCA do FG obtido com a assimilação do IWV, com as melhorias do modelo e com a ação conjunta de ambas as implementações para o HS, RT e AS (valores em horas).

\begin{tabular}{|c|c|c|c|c|c|c|c|c|}
\hline \multirow{2}{*}{\multicolumn{2}{|c|}{ Implementações }} & \multicolumn{7}{|c|}{ Variáveis } \\
\hline & & \multirow{2}{*}{$\begin{array}{l}\text { IWV } \\
25.8\end{array}$} & \multirow{2}{*}{$\begin{array}{c}\text { UR } \\
925 \mathrm{hPa} \\
15.1\end{array}$} & \multirow{2}{*}{$\begin{array}{c}\text { UR } \\
850 \mathrm{hPa}\end{array}$} & \multirow{2}{*}{$\begin{array}{c}\text { UR } \\
700 \mathrm{hPa} \\
20.3\end{array}$} & \multirow{2}{*}{$\begin{array}{c}\mathrm{UR} \\
500 \mathrm{hPa} \\
11.1\end{array}$} & \multirow{2}{*}{$\begin{array}{c}\text { UR } \\
300 \mathrm{hPa}\end{array}$} & \multirow{2}{*}{$\begin{array}{c}\mathrm{UR} \\
250 \mathrm{hPa} \\
11.6\end{array}$} \\
\hline \multirow{3}{*}{$\begin{array}{c}\text { Assimilação } \\
\text { do IWV (no } \\
\text { T126L28) }\end{array}$} & HS & & & & & & & \\
\hline & RT & 34.8 & 28.1 & 38.9 & 27.6 & 15.4 & 15.8 & 19.8 \\
\hline & AS & 30.0 & 14.4 & 20.6 & 16.3 & 8.9 & 7.7 & 10.2 \\
\hline \multirow{3}{*}{$\begin{array}{l}\text { Assimilação } \\
\text { do IWV (no } \\
\text { T213L42) }\end{array}$} & HS & 23.5 & 13.6 & 19.1 & 19.7 & 11.3 & 8.9 & 9.8 \\
\hline & $\mathrm{RT}$ & 32.3 & 22.0 & 29.4 & 26.9 & 14.4 & 13.0 & 15.2 \\
\hline & AS & 23.5 & 10.4 & 16.9 & 15.4 & 9.0 & 7.0 & 7.7 \\
\hline \multirow{3}{*}{$\begin{array}{l}\text { Melhorias do } \\
\text { modelo (não } \\
\text { assimilando } \\
\text { o IWV) }\end{array}$} & HS & 10.1 & 13.0 & 13.9 & 16.2 & 2.4 & 1.0 & -0.6 \\
\hline & RT & 10.5 & 8.9 & 13.9 & 9.0 & 3.1 & 2.2 & 1.1 \\
\hline & AS & 15.5 & 7.2 & 7.4 & 5.9 & 0.0 & 0.0 & 0.0 \\
\hline \multirow{3}{*}{$\begin{array}{c}\text { Melhorias do } \\
\text { modelo } \\
\text { (assimilando } \\
\text { o IWV) }\end{array}$} & HS & 0.3 & 1.7 & 0.5 & 1.2 & 0.6 & 0.0 & 0.0 \\
\hline & RT & 0.3 & 0.0 & 0.0 & 1.0 & 0.1 & 0.0 & 0.0 \\
\hline & AS & 0.6 & 0.0 & 0.0 & 0.3 & 0.0 & 0.0 & 0.0 \\
\hline \multirow{3}{*}{$\begin{array}{l}\text { Melhorias do } \\
\text { modelo e } \\
\text { assimilação } \\
\text { do IWV }\end{array}$} & HS & 27.8 & 21.5 & 25.5 & 26.6 & 12.3 & 9.1 & 11.1 \\
\hline & RT & 38.1 & 27.3 & 35.2 & 33.2 & 16.3 & 15.4 & 18.7 \\
\hline & AS & 32.3 & 15.5 & 20.8 & 18.4 & 8.6 & 7.1 & 9.7 \\
\hline
\end{tabular}


- Omaior impacto ocorre na região tropical, seguido pelo impacto verificado na América do Sul e Hemisfério Sul.

Comrelaçãoao perfil de umidade relativa(Figuras $3 \mathrm{e} 4$ ), além dos padrõesobservadosassociadosaosvaloresdoIWV, destaca-setambémque:

- O impacto é positivo em todos os níveis de pressão avaliados. No entanto, ele tende a ser mais significativo na baixa troposfera (nos níveis de 925, 850 e $700 \mathrm{hPa}$ ) onde a concentração de umidade é maior;

- No nível de $850 \mathrm{hPa}$ o impacto é sutilmente maior do que os demais níveis avaliados.

Os resultados que tornam mais evidente a importância da assimilação do IWV, são as ampliações da extensão das previsões válidas e os ganhos no CCA da umidade do FG mostrados nas Tabelas 2 e 3, respectivamente. Para os valores do IWV, observa-se que na versão T126L28 essa ampliação é de 18 horas na região tropical e de 12 horas na América do Sul, e ao considerar a versão T213L42, essa ampliação é de 17 e 10 horas, respectivamente. Para o perfil de umidade relativa os valores observados que merecem destaques na versão T126L28, ao considerar a RT, são: 26 horas em 850 hPa e 16 horas em 700 hPa e 925 hPa. Na versão T213L42, o destaque deve ser dado para o nível de $700 \mathrm{hPa}$, nessa mesma região, onde a expansão observada (17 horas) foi maior que nos demais níveis. Com relação às melhorias do $\mathrm{FG}$, os ganhos nos valores do CCA foram de 35, 30 e 26 horas para o IWV na região tropical, América do Sul e Hemisfério Sul, respectivamente. Ao considerar o perfil de umidade relativa, destaca-se o ganho obtido em $850 \mathrm{hPa}$, o qual foi de 39 horas na região tropical e de 21 horas na América do Sul e Hemisfério Sul. Em uma comparação entre as diferentes versões avaliadas do modelo, tanto na ampliação da extensão das previsões válidas, quanto no ganho no CCA da umidade do FG, pode-se observar que o impacto da assimilação do IWV é sutilmente mais significativo na versão T126L28 do que na versão T213L42, e que, enquanto na primeira o impacto é maior no nível de $850 \mathrm{hPa}$, na segunda esse impacto é maior no nível de $700 \mathrm{hPa}$.

\subsection{Impacto das Melhorias do Modelo na Previsibilidade dos Campos de Umidade}

Oimpacto das melhorias do modelo (novas implementações e aumento da resolução) na previsibilidade da umidade apresenta os seguintes padrões:

- Quando o IWV não é assimilado, os benefícios gerados pelas melhorias do modelo são significativos ao longo de todo o período de integração, impactando tanto na expansão da extensão das previsões válidas, como na melhoria do campo de umidade do FG.

- Quando o IWV é assimilado, o impacto tende a ser maior quanto maior o tempo de integração do modelo.
Enquanto para as previsões de 6 horas o ganho no CCA é praticamente nulo (veja Tabela 3), para previsões de 114 horas o aumento do CCA pode chegar a $8 \%$. Uma consequência desse padrão é que, ao contrário do impacto somente da assimilação do IWV, o impacto das melhorias do modelo quando o IWV é assimilado, gera maior expansão da extensão das previsões válidas do que melhoria no campo de umidade do FG.

- Na comparação entre as três regiões avaliadas, observa-se que: enquanto o impacto das melhorias do modelo, quando o IWV não é assimilado, apresenta resultados mais significativos na RT e AS. Quando o IWV é assimilado esse padrão não é observado.

Com relação ao perfil de umidade relativa (Figuras 3 e 4) os padrões observados são:

- Independentemente se o IWV é assimilado ou não, o impacto é positivo na baixa troposfera e é sutilmente negativo nos níveis de 300 e $250 \mathrm{hPa}$. Quando os valores do IWV são assimilados, há dois casos em que o impacto observado é inverso a esse padrão. O primeiro é com relação aos níveis mais elevados ao considerar todo o HS, e o segundo se refere ao nível de $850 \mathrm{hPa}$ ao considerar a região tropical. Na América do Sul, nos níveis mais elevados, observa-se que ao assimilar o IWV e após 90 horas de integração, há um impacto positivo, porém os valores nessas circunstâncias são menores do que 0.6;

- No nível de 700 hPa, com ou sem a assimilação do IWV, o impacto é positivamente maior do que nos demais níveis avaliados.

Com relação aos valores apresentados nas Tabelas 2 e 3, observa-se que ao assimilar o IWV o ganho no CCA da umidade do FG gerado pelas melhorias presentes no modelo é praticamente nulo (valores menores do que 2 horas), enquanto que na expansão da extensão das previsões válidas há alguns resultados que merecem destaques. Esse é o caso da expansão da extensão do período das previsões válidas para a variável IWV, a qual foi de 6 horas para a RT e AS. Com relação ao perfil da umidade relativa destacam-se as expansões observadas em $700 \mathrm{hPa}$ no Hemisfério Sul (21 horas), região tropical (15 horas), e em 925 hPa na América do Sul (10 horas). No entanto, quando os valores do IWV não são assimilados, o impacto das melhorias presentes no modelo é significativo, tanto na expansão das previsões válidas quanto no ganho no CCA da umidade do FG. Para a umidade relativa em $925 \mathrm{hPa}$ na AS é observado um valor de 25 horas na expansão das previsões válidas. Para os valores do IWV nessa mesma região a expansão das previsões válidas chega a 9 horas e o ganho no CCA do FG é de 16 horas. De forma geral, observa-se que o impacto das melhorias do modelo nas primeiras horas de integração é mais significativo quando os valores do IWV não são assimilados, e menos quando se assimila os valores do IWV. 


\subsection{Impacto da Ação Conjunta da Assimilação do IWV e das Melhorias do Modelo na Previsibilidade dos Campos de Umidade}

Como essa análise é uma combinação das duas anteriores, os padrões nelas observados estão aqui presentes, sendo que o impacto da assimilação do IWV predomina nas primeiras horas de previsão, enquanto que nas previsões de médio para longo prazo predomina o impacto das melhorias do modelo. Os padrões que podem ser identificados na variável IWV (Figura 2) são:

- O impacto é significativo ao longo de todas as previsões avaliadas, maior nas primeiras horas de integração do modelo, mas nunca tendendo a zero, como observado em algumas análises anteriores. Em consequência disso o impacto é significativo, tanto na expansão da extensão das previsões válidas, quanto no ganho no CCA do FG (veja Tabelas 2 e 3);

- O maior impacto ocorre na região tropical, seguido pelo impacto verificado na América do Sul e Hemisfério Sul. Com relação ao perfil de umidade relativa na região tropical (Figura 3) observa-se que:

- O impacto é positivo em todos os níveis avaliados, sendo mais significativo na baixa troposfera. Nos níveis de 850, 300 e $250 \mathrm{hPa}$, nos quais foi observado um sutil impacto negativo das melhorias do modelo, o impacto positivo da assimilação do IWV foi maior, de tal forma que o saldo final foi positivo;

- Em 700 hPa observa-se que o impacto foi mais significativo.

- Na América do Sul (Figura 4) observa-se que:

- O impacto é positivo nas primeiras horas de previsão para todos os níveis avaliados, mas para os níveis 300 e 250 hPa há intervalos de integração do modelo (entre 30-84 horas de integração e de 42-90 horas, respectivamente) em que ele se torna negativo.

- O resultado positivo é mais expressivo em $925 \mathrm{hPa}$.

Em uma análise dos valores apresentados nas Tabelas 2 e 3, é possível observar que tanto na expansão da extensão previsões válidas, como no ganho do CCA do FG, o benefício da ação conjunta da assimilação do IWV, e das melhorias no modelo, é maior do que os benefícios dessas implementações isoladamente. Nas previsões do IWV as expansões chegam a 12 horas no Hemisfério Sul, 18 horas na América do Sul e de 25 horas (maior que um dia) para a região tropical. Para os valores da umidade relativa, destacam-se o nível de $700 \mathrm{hPa}$ no Hemisfério Sul e na região tropical, onde as expansões das extensões das previsões válidas foram de 26 horas e 31 horas, respectivamente. Já na América do Sul a maior expansão (21 horas) foi observada em $925 \mathrm{hPa}$. Com relação ao ganho do CCA no FG para a variável IWV, valores de 28, 32 e 38 horas são observados para o Hemisfério Sul, América do Sul e região tropical, respectivamente. Para a umidade relativa, os valores foram significativos para todos os níveis avaliados. $\mathrm{O}$ maior valor foi observado em $700 \mathrm{hPa}$ na região tropical (33 horas de ganho) e o menor é observado no nível de 300 hPa na América do Sul (7 horas de ganho).

De forma geral, observa-se que a combinação das duas práticas gera um impacto positivo bastante significativo ao considerar as previsões do IWV, bem como a umidade relativa nos níveis em que a concentração de umidade é elevada ( 925 , 850 e 700 hPa). Na Figura 5 essas variáveis são utilizadas para mostrar em termos de ganho percentual o impacto da combinação das duas implementações para as três diferentes regiões avaliadas. Essa figura mostra que o ganho no CCA no IWV (Figura 5a) em termos percentuais é de $27 \%$ nas primeiras 6 horas na RT e é acima de $9 \%$ em todo o período de integração e para qualquer uma das regiões estudadas. Embora o ganho na umidade relativa em $850 \mathrm{hPa}$ e $925 \mathrm{hPa}$ (Figuras $5 \mathrm{~b}$ e 5c) na RT seja bastante elevado nas primeiras horas ( $40 \%$ e $25 \%$, respectivamente), o mesmo decai em função do tempo de integração do modelo e torna-se após 78 horas, pior do que os valores observados nas demais regiões avaliadas. No nível de $700 \mathrm{hPa}$ (Figura 5d) observa-se que o impacto em termos percentuais torna-se maior, quanto maior for o tempo de integração do modelo, independente da região considerada. Para a RT, onde o ganho percentual é de 26\% nas primeiras 6 horas, o mesmo chega a 36\% após 114 horas de integração do modelo.

\section{CONCLUSÕES E COMENTÁRIOS FINAIS}

Foram realizados quatro experimentos visando avaliar o impacto da assimilação de valores do IWV e das melhorias implementadas do MCGA na previsibilidade dos campos de umidade gerados pelo CPTEC/INPE. No primeiro experimento foi utilizada a versão do modelo sem a implementação das melhorias, na resolução T126L28 e sem assimilar o IWV, e no segundo experimento a mesma versão do modelo foi utilizada, mas com assimilação do IWV. No terceiro experimento foi utilizada uma versão do modelo em que novas implementações foram feitas, na resolução T213L42, mas sem a assimilação do IWV. Enquanto que no quarto e último foi utilizada essa mesma nova versão, mas com assimilação do IWV. A configuração dos experimentos permitiu avaliar tanto o impacto isolado da assimilação do IWV nas diferentes versões do modelo, quanto o impacto isolado das melhorias do modelo com e sem a assimilação do IWV, bem como o impacto combinado dessas duas implementações.

O impacto da assimilação do IWV, considerando ou não as novas implementações, é bastante significativo nas primeiras horas de previsão, decaindo com o tempo de integração do 


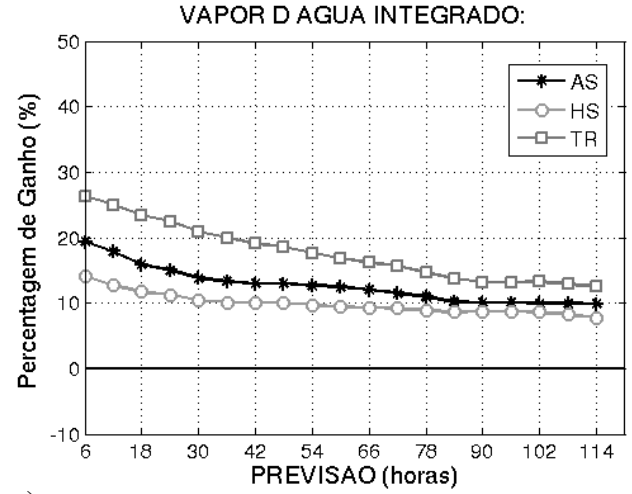

a)

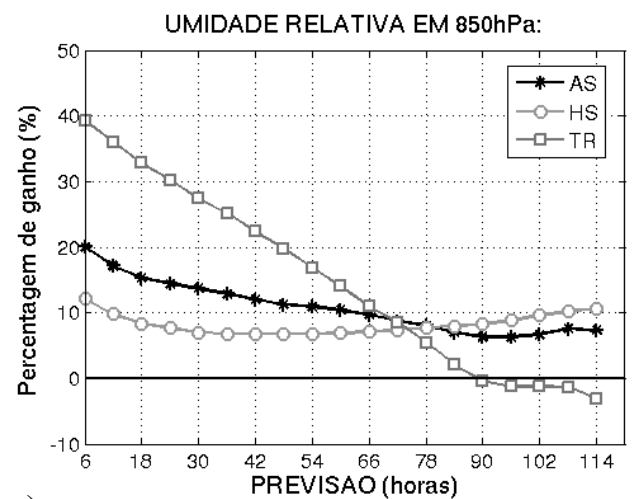

c)

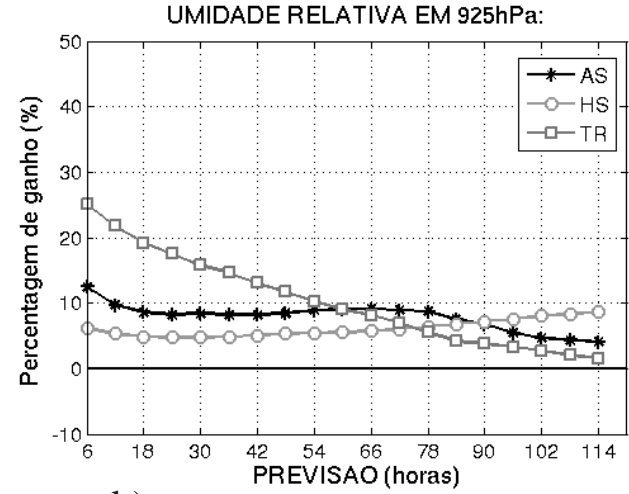

b)

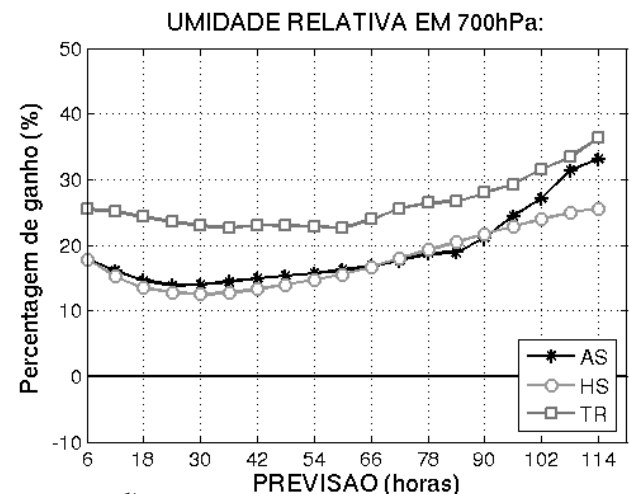

d)

Figura 5 - Percentuais de ganho nos valores do CCA gerados pela combinação da assimilação do IWV e das melhorias no modelo para as previsões do (a) IWV e da umidade relativa em (b) 925, (c) 850 e (d) $700 \mathrm{hPa}$.

modelo. Como consequência o impacto é maior no ganho do CCA do FG do que na expansão da extensão das previsões válidas. Embora o impacto seja sensível em todas as regiões avaliadas, na região tropical ele é mais significativo. Nessa região a expansão da extensão das previsões válidas do IWV chegou a 18 horas, e o ganho em horas do CCA do FG foi de 35 horas. Ao considerar as diferentes versões do modelo utilizadas, os resultados mostram que o impacto da assimilação do IWV é mais significativo na versão sem as novas implementações. Esse resultado é um indicativo de que o uso de modelos mais sofisticados tende a amenizar a deficiência na disponibilidade de observações de umidade.

O impacto isolado das melhorias presentes no modelo (novas implementações e aumento da resolução), quando se assimila valores do IWV é significativo depois de algum tempo de integração do modelo. Nesse caso, enquanto que o ganho no CCA do FG é praticamente nulo, a expansão da extensão das previsões válidas foi de, em média, 6 horas para o IWV. Quando os valores do IWV não são assimilados observa-se que o impacto das melhorias presentes no modelo é significativo ao longo de todo o tempo de integração do modelo, com benefícios tanto na expansão das previsões válidas como no ganho no CCA da umidade do FG. Para os valores do IWV na AS, a expansão das previsões válidas observada foi de 9 horas e o ganho no CCA do FG foi de 16 horas. Considerando apenas as primeiras horas de integração, observa-se que o impacto das melhorias do modelo é mais significativo quando os valores do IWV não são assimilados. Esse é um forte e importante indicativo de que para a previsibilidade dos campos de umidade, a assimilação do IWV deve ser privilegiada mesmo quando não se tem à disposição modelos aprimorados.

No experimento em que as duas práticas foram adotadas, seus impactos isolados são combinados e o resultado é significativo ao longo de todas as previsões avaliadas. Esse impacto foi mais intenso nas primeiras horas de integração do modelo, onde a assimilação do IWV é predominante. Por consequência, observa-se que o impacto é substancial, tanto na expansão da extensão das previsões válidas, quanto no ganho no CCA do FG. Esse impacto é mais significativo nos valores do IWV e nos níveis da umidade relativa onde se concentra a umidade (925, 850 e 700 hPa). Embora seja observado nas três regiões avaliadas, na região tropical o impacto foi mais expressivo. Nessa região a expansão 
das previsões válidas do IWV foi de 25 horas e o ganho no CCA do FG foi de 38 horas. Em termos percentuais observou-se que o ganho no CCA nas primeiras horas de integração do modelo na RT foi de $27 \%$ e $40 \%$ para as previsões do IWV e da umidade relativa em $850 \mathrm{hPa}$, respectivamente.

Com a ampliação da capacidade computacional do CPTEC, que se encontra em curso com a aquisição de mais um novo supercomputador, tornará viável a realização de novos experimentos com períodos mais longos de assimilação, os quais permitirão acessar o impacto dessas implementações sazonalmente. Resultados apresentados por Gutman et al. (2004) e Smith et al. (2007), mostram que o impacto da assimilação do IWV é mais significativo no inverno, quando as mudanças na umidade são dominadas por sistemas de tempo de escala sinótica, do que no verão. Outros trabalhos que objetivam avaliar o impacto das novas implementações do modelo na previsibilidade da precipitação, encontram-se em fase de desenvolvimento no grupo de modelagem do CPTEC/INPE (Figueroa et al., 2006; Silvio Nilo Figueroa, 2009, comunicação pessoal). O mesmo ocorre no grupo de assimilação desse mesmo centro no que se refere à avaliação do impacto da assimilação do IWV na previsibilidade da precipitação sobre a América do Sul (Sapucci et al., 2010). Nesse trabalho é contemplada também uma avaliação com relação aos valores observados, tanto de umidade como de outras variáveis atmosféricas.

\section{AGRADECIMENTOS.}

Os autores agradecem as pessoas que direta ou indiretamente estiveram envolvidas com as modificações implementadas na nova versão do modelo de PNT do CPTEC, em especial o Dr. José Paulo Bonatti e o Dr. Jairo Panetta, e seus respectivos grupos. Especiais agradecimentos à Msc. Simone S. Tomita pelas relevantes contribuições na adaptação do PSAS à nova versão do modelo de PNT do CPTEC. Os autores agradecem também ao CNPq (Conselho Nacional de Desenvolvimento Científico e Tecnológico) (processos CNPq ${ }^{\circ}$ 475523/2007-5 e n 476129/2006-0) e à FAPESP (Fundação de Amparo a Pesquisa do Estado de São Paulo) (processo FAPESP $n^{\circ} 2006 / 04008-2$ ) pelo apoio financeiro a essa pesquisa.

\section{REFERÊNCIAS BIBLIOGRÁFICAS}

ANTHES, R. A.; BERHARDT, P. A.; CHEN, Y.; CUCURULL, L.; DYMOND, K. F.; ECTOR, D.; HEALY, S. B.; HO, S.P.; HUNT, D. C.; KUO, Y.-H.; LIO, H.; MANNING, K.; MCCORMICK, C.; MEEHAN, T. K.; RANDEL, W. J.; ROCKEN, C.; SCHREINER, W. S.; SOKOLOVSKIY, S. V.;
SYNDERGAARD, S.; THOMPSON, D.; TRENBERTH, K. E.; WEE,T.-K.;YEN,N.L.;ZEND,Z.TheCOSMIC/FORMOSAT-3 Mission, B. Am. Meteorol. Soc., 89, 313-333, 2008. BEVIS, M. G., SUSINGER S.; HERRING T.; ROCKEN C.; ANTHES R. A.; WARE R. H. GPS meteorology: remote of atmospheric water vapor using the Global Positioning System. Journal of Geophysical Research, 97, 15.787-15.801, 1992. CAVALCANTI, I. F. A.; MARENGO, J. A.; SATYAMURTY, P.; NOBRE, C. A.; TROSNIKOV, I.; BONATTI, J.P; MANZI, A O.; TARASOVA, T.; PEZZI, L.P.; D’ALMEIDA, C.; SAMPAIO, G.; CASTRO, C.C.; SANCHES, M. B.; CAMARGO, H. Global climatological features in a simulation using CPTEC/COLA AGCM. J. Climate, 15, 2965-2988, 2002.

CHOU, M. D.; SUAREZ, M. J. A Solar Radiation Parameterization (CLIRAD-SW). NASA Technical Memorandum no. 104606, v. 15, 48 pp., 1996.

COHN, S. E.; DA SILVA, A.; GUO, J.; SIENKIEWICZ, M.; LAMICH, D. Assessing the effects of the data selection with the DAO physical-space statistical analysis system. Mon. Wea. Rev., v.126, p. 2913-2926, 1998.

FIGUEROA, S. N.; TARASOVA, T. A.; BARBOSA, H. M. J.; BONATTI, J. P.; SILVA DIAS P. L. The impact of cumulus and radiation parameterization schemes on Southern Hemisphere summer climate simulate by CPTEC Atmospheric General Circulation Model. In: 8 INTERNATIONAL CONFERENCE ON SOUTHERN HEMISPHERE METEOROLOGY AND OCEANOGRAPHY, Anais... Foz do Iguaçu. 2006.

GAL-CHEN, T.; SCHMIDT, B.D.; UCCELLINI, L.W. Simulation Experiments for Testing the Assimilation of Geostationary Satellite Temperature Retrievals into a Numerical Prediction Model. Mon. Wea. Rev., 114, 1213-1230, 1986.

GRELL, G. A. Prognostic Evaluation of Assumptions Used by Cumulus Parameterizations Monthly Weather Review, 121: 764-787, 1993.

GUTMAN, S. I.; SAHM, S.; BENJAMIN, S. G.; SCHWARTZ, B. E.; HOLUB, K.; STEWART J. Q.; SMITH, T. L. Rapid retrieval and assimilation of ground based GPS precipitable water observations at the NOAA forecast systems laboratory: Impact on weather forecasts, Journal of the Meteorological Society of Japan, 82(1B), 351-360, 10.2151/jmsj.2004.351, 2004.

HOLTON, I. R., 1992: An introduction to dynamic meteorology. $3^{\text {rd }}$ ed. Academic Press, 507 pp.

HOU, A.Y.; ZHANG, S.Q.; DA SILVA, A.M.; OLSON, W.S. Improving Assimilated Global Datasets Using TMI Rainfall and Columnar Moisture Observations. J. Climate, 13, 4180-4195, 2000. 
KALNAY, E. Atmospheric Modeling, Data Assimilation, and Predictability. Cambridge University Press, Cambridge. 364 p, 2003.

KINTER, J.L. et al The COLA Atmosphere Biosphere General Circulation Model. Volume1: Formulation. Center for Ocean LandAtmosphere Studies. ReportN.51. Calverton, USA, 1997.

KRISHNAMURTI, T. N.; RAJENDRAN, K.; KUMAR, T. S. V.; LORD, S.; TOTH, Z.; ZOU, X.; COCKE, S.; AHLQUIST, J. E.; NAVON, I. M. Improved Skill for the Anomaly Correlation of Geopotential Heights at $500 \mathrm{hPa}$, Mon. Weather Rev., v. 131, p. 1082-1102, 2003.

KUO, Y. H.; GUO, Y. R.; WESTWATER, E. R. Assimilation of Precipitable Water Into Mesoscale Numerical Model. Mon. Wea. Rev., 121, 1215-1238, 1993.

LEDVINA, D. V.; PFAENDTNER, J. Inclusion of SSM/I total precipitable water estimates into the GEOS-1 data assimilation system. Mon. Wea. Rev., 123, 3003-3015, 1995.

RANDEL, D. L., VONDER HAAR, T. H., RINGERUD, M. A., STEPHENS, G. L., GREENWALD, T. J., COMBS, C. L. A new global water vapor dataset. Bulletin of the American Meteorological Society, Volume 77, Number 6. 1996.

REALE, A L. NOAA operational sounding products for advanced-TOVS, NOAA Tech. Rep. NESDIS 107, 29pp. U.S. Dep. of Commer. Washington, D. C., 2002.

SAPUCCI, L. F.; MACHADO, L.A. T.; SILVEIRA, R. B.; FISCH, G.; MONICO, J. F. G. Analysis of relative humidity sensors at WMO radiosonde intercomparison experiment in Brazil. J. Atmos. Oceanic Technol., v. 22, n.6, p.664-678, 2005. SAPUCCI, L. F.; MACHADO, L. A. T.; MONICO, J. F. G.; PLANA-FATTORI, A. Integrated water vapor quantification in Amazonian regions. J. Atmos. Oceanic Technol, v. 24, n.11, p.1880-1894, 2007.
SAPUCCI, L. F., et al. Impact analysis of the inclusion of IWV estimates from AIRS/AMSU and SSM/I sensors into the CPTEC/INPE's global data assimilation system. Submitted at International Journal Remote Sensing. 2010.

SMITH, T. L.; BENJAMIM, S. G.; SCHWARTZ, B. E.; GUTMAN, S. I. Using GPS-IPW in a 4-D data assimilation system. Earth Planets Space, v.52, p. 921-926, 2000.

SMITH, T. L.; BENJAMIM, S. G.; GUTMAN, S. I.; SAHM, S. Short-Range Forecast Impact from Assimilation of GPSIPW Observations into the Rapid Update Cycle. Monthly Weather Review, Vol. 135, N.8, pp. 2914-2930, 2007.

SOUZA, E. P. Estudo Teórico e Numérico da Relação entre Convecção e Superfícies Heterogêneas na Região Amazônica. 1999. 121 f. Tese (Doutorado em Meteorologia). Universidade de São Paulo, 1999.

STAUFFER, D.R.; SEAMAN N.L. Use of Four-Dimensional Data Assimilation in a Limited-Area Mesoscale Model. Part I: Experiments with Synoptic-Scale Data. Mon. Wea. Rev., 118, 1250-1277, 1990.

SUSSKIND, J.; BARNET, C.; BLAISDELL, J. Retrieval of atmospheric and surface parameters from AIRS/AMSU/ HSB data in the presence of clouds. IEEE Transactions on Geoscience and Remote Sensing, v.41, n.2, p. 390409, 2003.

TOMITA, S. S.; KUBOTA, P. Y.; BONATTI, J. P.; HERDIES, D. L. Testes de Sensibilidade do Novo Modelo Global MCGA/ MPI - CPTEC/INPE Usando Diferentes Parametrizações Físicas. In. XIV Congresso Brasileiro de Meteorologia. 2006. Florianópolis-SC. Anais..., Florianópolis, Brasil, 